Accepted to The international Conference on Acoustics, Speech, \& Signal Processing (ICASSP), Toronto, Canada, 6-11 June 2021.

\title{
ENERGY EFFICIENCY OPTIMIZATION TECHNIQUE FOR SWIPT-ENABLED MULTI-GROUP MULTICASTING SYSTEMS WITH HETEROGENEOUS USERS
}

\author{
Sumit Gautam, Symeon Chatzinotas, and Björn Ottersten \\ Interdisciplinary Centre for Security, Reliability and Trust, University of Luxembourg, Luxembourg.
}

\begin{abstract}
We consider a multi-group (MG) multicasting (MC) system wherein a multi-antenna transmitter serves heterogeneous users capable of either information decoding (ID) or energy harvesting $(\mathrm{EH})$, or both. In this context, we investigate a precoder design framework to explicitly serve the ID and EH users categorized within certain MC and EH groups. Specifically, the ID users are categorized within multiple MC groups while the EH users are a part of single (last) group. We formulate a problem to optimize the energy efficiency in the considered scenario under a quality-of-service (QoS) constraint. An algorithm based on Dinkelback method, slack-variable replacement, and second-order conic programming (SOCP)/semi-definite relaxation (SDR) is proposed to obtain a suitable solution for the above-mentioned fractionalobjective dependent non-convex problem. Simulation results illustrate the benefits of proposed algorithm under several operating conditions and parameter values, while drawing a comparison between the two proposed methods.
\end{abstract}

Index Terms - Fractional optimization, Multi-group (MG) Multicasting (MC), Precoding, SDR, SOCP, SWIPT.

\section{INTRODUCTION}

The emerging fifth generation (5G)-and beyond wireless communication systems and recent trends in the Internet-ofThings (IoTs) emphasize on an increased throughput with ultra-low latency [1]. To realize this prospect, exploiting the trade-off between the system capacity and power consumption at the concerned nodes becomes crucial, considering the complex hardware designs and hefty device applications. In this context, efficient power optimization and energy harvesting (EH) methods may be devised to tackle the abovementioned challenges [2,3]. Moreover, it is also important develop mechanism(s) that consider the co-existential aspect of heterogeneous device types like information decoding (ID) specific, explicit to energy harvesting $(\mathrm{EH})$, and the ones capable of performing both ID and EH concurrently.

In order to enhance the communication efficiency of wireless systems while additionally facilitating the EH mechanism, methods such as precoding for Multi-group (MG) Multicast (MC) and simultaneous wireless information and

Thanks to the Luxembourg National Research Fund (FNR), for funding this work under the FNR-FNRS bilateral - InWIP-NET (R-AGR-0700-10-X). The authors thank Mr. Ashok Bandi and Mr. Aakash Arora for their help in the form of fruitful discussions. power transmission (SWIPT) have shown immense potential $[4,5,6]$. In this context, the benefits of MG-MC precoding were demonstrated in $[7,8,9]$, without the consideration of SWIPT. Regarding the EH in SWIPT systems, the separated architecture (SA)-based receiver architecture seems promising over the power-splitting (PS) and time-switching (TS)-based counterparts, primarily due to the hardware concerns pertaining the multiple variable optimizations in PS and TS [10]. Several works discuss joint information and energy transfer in the MG-MC scenario, however, with an assumption of a linear EH module [11, 12]. A framework to investigate PS-based SWIPT Multicasting was presented in [13] with individual Quality-of-Service (QoS) constraints to meet the demands of energy sustainable Internet-of-Things (IoT). Noticeably, these works do not consider coexistence of different user types within the MG-MC.

More recently, the authors investigated the performances of three precoding schemes for MG-MC systems incorporating the heterogeneous user types $[2,3,14]$. Specifically, the problems of total transmit power minimization $[2,3]$ and maximization of the sum-signal-to-interference-plus-noise ratio (SINR) and minimum of the SINR [14] were addressed. In this paper, we consider the problem of MG-MC precoding to minimize the ratio of total transmit energy to the sum-harvested energy at the intended devices. The transmit source is assumed to be equipped with an array of antennas which serves multiple users with the aid of beamforming through adequate precoders. In this context, we investigate this problem by considering the aspect of co-existence of three different types of users. The non-convexity of the aforementioned problem lead to intractability. We propose a tri-level alternating parameter (TAP) optimization based iterative algorithm comprised of the Dinkelback method, slackvariable replacement, and second-order cone programming $(\mathrm{SOCP}) /$ semi-definite relaxation (SDR) techniques.

Further sections of this paper are organized as follows. Section 2 provides an introduction to the system model. The problem formulation and the proposed solution are presented in Section 3. Numerical results are shown in Section 4, followed by concluding remarks in Section 5 .

Notation: Bold face lower case and upper case characters denote column vectors and matrices, respectively. The operators $(\cdot)^{\dagger}$ and $|\cdot|$ correspond to the conjugate transpose and the absolute value, respectively. The real and imaginary parts of a complex value are defined as $\Re(\cdot)$ and $\Im(\cdot)$, respectively. 
Accepted to The international Conference on Acoustics, Speech, \& Signal Processing (ICASSP), Toronto, Canada, 6-11 June 2021.

\section{SYSTEM MODEL}

We consider a system model consisting of an $M$-antenna equipped transmitter that serves $L$ devices $\left(\mathcal{D}_{1}, \ldots, \mathcal{D}_{L}\right)$. The devices are assumed to be of heterogeneous types, specifically, the users able to decode information, users able to harvest energy, and users capable of simultaneously performing both, respectively. The latter are assumed to be equipped with two separate RF chains designed exclusively to carry out the desired operations of ID and EH. We aim to design optimal precoders in a system capable of serving the MC and EH group of users distinctly, yet concurrently. Specifically, we assume $G \mathrm{MC}$ information groups and an additional group devoted to $\mathrm{EH}$ specific users. Thus, we target design of $(G+1)$ precoders.

Let $\mathcal{G}_{k}$ denote the $k^{\text {th }}$ multicast group of users. We assume known MC groups and the last EH group in this work. Each user adheres to listen only the MC specific for the group where corresponding user is present i.e., $\mathcal{G}_{k} \cap \mathcal{G}_{\ell}=\varnothing$, $\forall k, \ell=\{1, \ldots, G+1\}$ and $k \neq \ell$; whereas in case of $\mathrm{EH}$, the user harvests energy using all the possible multicast signals. The antenna array at the transmitter emits the signal $\mathbf{y}_{\tau}(t)=\sum_{k=1}^{G+1} \mathbf{w}_{k} s_{k}(t)$, where $\mathbf{w}_{k}$ is the related $M \times 1$ complex precoding weight vector for the users in group $\mathcal{G}_{k}$, and $s_{k}(t)$ is the corresponding information and/or energy signal, such that for each group $\left\{s_{k}(t)\right\}_{k=1}^{G+1}$ are mutually uncorrelated to each other with zero mean and unit variance, $\sigma_{s_{k}}^{2}=1$. The total transmitted energy is given by $\sum_{k=1}^{G+1} \mathbf{w}_{k}^{\dagger} \mathbf{w}_{k}$.

The received signal at the $x^{\text {th }}$ user is given by $y_{r, x}(t)=$ $\mathbf{h}_{x}^{\dagger} \mathbf{y}_{\tau}(t)+n_{r, x}(t)$, where $\mathbf{h}_{x}$ is the $M \times 1$ conjugated channel vector for the corresponding receiver and $n_{r, x}(t)$ is the additive zero mean Gaussian noise at the corresponding $x^{\text {th }}$ user's receiving antenna equipment with a noise variance of $\sigma_{r, x}^{2}$. The source signals are uncorrelated with $n_{r, x}(t)$. The information decoding unit of the $x^{\text {th }}$ receiver equipment is expressed as : $y_{d, x}(t)=\left(\mathbf{h}_{x}^{\dagger} \mathbf{y}_{\tau}(t)+n_{r, x}(t)\right)+n_{d, x}(t)$, where $n_{d, x}(t)$ is the additional zero-mean Gaussian noise with a variance of $\sigma_{d, x}^{2}$ incurred due to the circuitry and other relevant operations at the ID block of the $x^{\text {th }}$ receiver. The signal dedicated for EH block of the $x^{\text {th }}$ receiver is: $y_{e, x}(t)=\mathbf{h}_{x}^{\dagger} \mathbf{y}_{\tau}(t)+$ $n_{r, x}(t)$. In this regard, we define the harvested energy at the receiver according to the non-linear EH model as follows

$$
\widehat{E}_{x}=\frac{\mathcal{E}^{\prime}}{1-\nu} \cdot\left(\frac{1}{1+e^{\left(-\lambda\left(\sum_{k=1}^{G+1}\left|\mathbf{w}_{k}^{\dagger} \mathbf{h}_{x}\right|^{2}\right)+\lambda \mu\right)}}-\nu\right),
$$

where $\nu \triangleq \frac{1}{1+\exp (\lambda \mu)}$, the constant $\mathcal{E}^{\prime}, \lambda$ and $\mu$ are related to the non-linear EH module $[15,16]$. We assume normalized time slots to use the terms power and energy interchangeably.

\section{ENERGY EFFICIENCY OPTIMIZATION}

In this section, we formulate the optimization problem to minimize the ratio of the overall transmitted energy by the transmitter to the sum-harvested energy at the intended de- vices (termed as inverse energy efficiency), subjected to minimum SINR constraints at the corresponding users/groups. The overall optimization problem to ensure the co-existence of the three user types in the MG-MC precoding scheme can subsequently be written in its mathematical form as follows

$$
\begin{aligned}
(P 1): \min _{\left\{\mathbf{w}_{i}\right\}_{i=1}^{G+1}} & \frac{\sum_{i=1}^{G+1} \mathbf{w}_{i}^{\dagger} \mathbf{w}_{i}}{\sum_{\forall j \in \mathcal{G}_{G+1}} \widehat{E}_{j}} \\
\text { s.t. } & (C 1): \frac{\left|\mathbf{w}_{y}^{\dagger} \mathbf{h}_{x}\right|^{2}}{\sum_{z \neq y}\left|\mathbf{w}_{z}^{\dagger} \mathbf{h}_{x}\right|^{2}+\sigma_{r, x}^{2}+\sigma_{d, x}^{2}} \geq \gamma_{x}, \\
& \forall x \in \mathcal{G}_{y}, \forall y \in\{1, \ldots, G\}, \forall z \in\{1, \ldots, G+1\},
\end{aligned}
$$

where $\gamma_{x}$ is the SINR threshold at the $x^{\text {th }}$ user. It is clear that the formulated problem $(P 1)$ is not convex and hence intractable. In order to tackle the fractional objective in $(P 1)$, we employ the Dinkelback method, as described below

$$
\begin{aligned}
(P 2): \min _{\left\{\mathbf{w}_{i}\right\}_{i=1}^{G+1}, \alpha} & \sum_{i=1}^{G+1} \mathbf{w}_{i}^{\dagger} \mathbf{w}_{i}-\alpha\left(\sum_{\forall j \in \mathcal{G}_{G+1}} \widehat{E}_{j}\right) \\
\text { s.t. } & (C 1),
\end{aligned}
$$

where $\alpha$ is the introduced auxiliary variable required to carry out the Dinkelback process. Further, we observe that $\widehat{E}_{j}$ is a non-linear function and its presence in the objective of $(P 2)$ introduces an intractability. Therefore, we make use of the slack-variable replacement technique to further transform $(P 2)$ into a simpler form, expressed as

$$
\begin{aligned}
(P 3): \min _{\substack{\left\{\mathbf{w}_{i}\right\}_{i=1}^{G+1}, \alpha,\left\{\xi_{j}\right\}_{\forall j \in \mathcal{G}_{G+1}}}} \sum_{i=1}^{G+1} \mathbf{w}_{i}^{\dagger} \mathbf{w}_{i}-\alpha\left(\sum_{\forall j \in \mathcal{G}_{G+1}} \xi_{j}\right) \\
\text { s.t. }(C 1), \quad(C 2): \widehat{E}_{j} \geq \xi_{j}, \forall j \in \mathcal{G}_{G+1},
\end{aligned}
$$

where $\xi_{j}$ is the slack variable corresponding to the $j^{\text {th }}$ user present in the $\mathcal{G}_{G+1}{ }^{\text {th }}$ group comprising the EH users. However, the presence of $\widehat{E}_{j}$ in $(C 2)$ of $(P 3)$ would continue to provide hindrance in seeking a suitable solution, due to the pertaining non-linearity. Thus, we re-express $(P 3)$ to provide more tractability, as represented in the following

$$
\begin{aligned}
&(P 4): \min _{\substack{\left\{\mathbf{w}_{i}\right\}_{i=1}^{G+1}, \alpha,\left\{\xi_{j}\right\}_{\forall j \in \mathcal{G}_{G+1}}}} \sum_{i=1}^{G+1} \mathbf{w}_{i}^{\dagger} \mathbf{w}_{i}-\alpha\left(\sum_{\forall j \in \mathcal{G}_{G+1}} \xi_{j}\right) \\
& \text { s.t. }(C 1),(C 2): \mathcal{A}\left(\mathbf{w}_{j}\right) \geq \xi_{j} \mathcal{B}\left(\mathbf{w}_{j}\right), \forall j \in \mathcal{G}_{G+1},
\end{aligned}
$$

where $\mathcal{A}\left(\mathbf{w}_{j}\right)=\mathcal{E}^{\prime}\left(1-\nu\left(1+e^{\left(-\lambda\left(\sum_{i=1}^{G+1}\left|\mathbf{w}_{i}^{\dagger} \mathbf{h}_{j}\right|^{2}\right)+\lambda \mu\right)}\right)\right)$ and $\mathcal{B}\left(\mathbf{w}_{j}\right)=(1-\nu)\left(1+e^{\left(-\lambda\left(\sum_{i=1}^{G+1}\left|\mathbf{w}_{i}^{\dagger} \mathbf{h}_{j}\right|^{2}\right)+\lambda \mu\right)}\right)$. In the current form, $(C 2)$ may be solved by iteratively computing the involved parameters after application of certain approximations/relaxations. Finally, the only remaining barrier in finding a solution of $(P 4)$ lies in the SINR constraint, i.e., $(C 1)$. In this context, we discuss two possibilities to seek a suitable solution for the intended parameters via second-order cone programming (SOCP) and semi-definite programming (SDP) followed by semi-definite relaxation (SDR), respectively, as presented in the following sections. 
Accepted to The international Conference on Acoustics, Speech, \& Signal Processing (ICASSP), Toronto, Canada, 6-11 June 2021.

\subsection{Second-order conic programming (SOCP)}

In this section, we aim at finding the solution of $(P 4)$ with the help of SOCP technique. The SOCP technique is considered to primarily tackle the non-convexity introduced by the SINR constraint in the formulated problem. In this vein, we split the SINR constraint $(C 1)$ (mentioned in $(P 1)$ - $(P 4)$ ), as follows

$$
\begin{aligned}
(C 1)_{a}: \mathbf{w}_{y}^{\dagger} \mathbf{h}_{x} \geq \sqrt{\gamma_{x}\left(\sum_{z \neq y}\left|\mathbf{w}_{z}^{\dagger} \mathbf{h}_{x}\right|^{2}+\sigma_{r, i}^{2}+\sigma_{d, i}^{2}\right)}, \\
(C 1)_{b}: \Re\left(\mathbf{w}_{y}^{\dagger} \mathbf{h}_{x}\right) \geq 0, \Im\left(\mathbf{w}_{y}^{\dagger} \mathbf{h}_{x}\right)=0, \forall x \in \mathcal{G}_{y}, \\
\forall y \in\{1, \ldots, G\}, \forall z \in\{1, \ldots, G+1\} .
\end{aligned}
$$

Regarding the non-linear EH constraint $(C 2)$ (in $(P 4))$, we observe the pertaining non-convexity in the constraint from the perspective of implementing the SOCP technique. In this context, we introduce new auxiliary variables $\left(\left\{\varsigma_{i}\right\}_{\forall i \in \mathcal{G}_{G+1}}\right)$ to relax the corresponding constraint as:

$$
\begin{array}{r}
(C 2)_{a}: \widetilde{\mathcal{A}}\left(\varsigma_{j}\right) \geq \xi_{j} \widetilde{\mathcal{B}}\left(\varsigma_{j}\right), \\
(C 2)_{b}: \sum_{i=1}^{G+1}\left|\mathbf{w}_{i}^{\dagger} \mathbf{h}_{j}\right|^{2} \geq \varsigma_{j}, \forall j \in \mathcal{G}_{G+1},
\end{array}
$$

where $\widetilde{\mathcal{A}}\left(\varsigma_{j}\right)=\mathcal{E}^{\prime}\left(1-\nu\left(1+e^{\left(-\lambda \varsigma_{j}+\lambda \mu\right)}\right)\right)$ and $\widetilde{\mathcal{B}}\left(\varsigma_{j}\right)=(1-$ $\nu)\left(1+e^{\left(-\lambda \varsigma_{j}+\lambda \mu\right)}\right)$. The still persisting non-convexity in (7) may be addressed by using the first order Taylor series approximation of $\left(\left|\mathbf{w}_{y}^{\dagger} \mathbf{h}_{x}\right|^{2}\right)_{m+1}$ around $\left(\left|\mathbf{w}_{y}^{\dagger} \mathbf{h}_{x}\right|\right)_{m}$, given by

$$
\left(\left|\mathbf{w}_{y}^{\dagger} \mathbf{h}_{x}\right|^{2}\right)_{m+1}=\left(\left(\left|\mathbf{w}_{y}^{\dagger} \mathbf{h}_{x}\right|\right)_{m}\right)^{2}+\mathcal{C}\left(\left|\mathbf{w}_{y}^{\dagger} \mathbf{h}_{x}\right|\right),
$$

where $\mathcal{C}\left(\left|\mathbf{w}_{y}^{\dagger} \mathbf{h}_{x}\right|\right)=2\left(\left|\mathbf{w}_{y}^{\dagger} \mathbf{h}_{x}\right|\right)_{m}\left(\left(\left|\mathbf{w}_{y}^{\dagger} \mathbf{h}_{x}\right|\right)_{m}-\left(\left|\mathbf{w}_{y}^{\dagger} \mathbf{h}_{x}\right|\right)_{m+1}\right)$, with $(m+1)$ denoting the $(m+1)^{\text {th }}$ (current) iteration [17]. In this vein, we re-express $(C 2)_{b}$ as follows:

$$
(C 2)_{b}^{\star}: \sum_{i=1}^{G+1}\left(\left(\left(\left|\mathbf{w}_{i}^{\dagger} \mathbf{h}_{j}\right|\right)_{m}\right)^{2}+\mathcal{C}\left(\left|\mathbf{w}_{i}^{\dagger} \mathbf{h}_{j}\right|\right)\right) \geq \varsigma_{j}, \forall j \in \mathcal{G}_{G+1} .
$$

It is noteworthy that in $(C 2)_{b}^{\star}$, the value of $\left(\left(\left|\mathbf{w}_{i}^{\dagger} \mathbf{h}_{j}\right|\right)_{m}\right)$ is dependent on the initial guess, while its value is considered as the previous solution obtained during the iterative process. Additionally, it is clear that sum of affine functions is an affine function and hence the convexity at the constraint in $(C 2)_{b}^{\star}$ is manageable via alternating parameter based optimization technique. Further, we make use an approximation: $\left|\mathbf{w}_{i}^{\dagger} \mathbf{h}_{j}\right|^{2}=\mathbf{w}_{i}^{\dagger} \mathbf{h}_{j} \mathbf{h}_{j}^{\dagger} \mathbf{w}_{i}$ (primarily because $\left|\mathbf{w}_{i}^{\dagger} \mathbf{h}_{j}\right|^{2} \geq 0$ ) to provide more tractability to the problem. Correspondingly, we re-write $(P 4)$ by incorporating the above-mentioned constraints pertaining the SOCP, in the following.

$$
\begin{aligned}
(P 5): \min _{\substack{\left\{\mathbf{w}_{i}\right\}_{i+1}^{G+1}, \alpha,\left\{\xi_{j}, \varsigma_{j}\right\}_{\forall j \in \mathcal{G}_{G+1}}}} \sum_{i=1}^{G+1} \mathbf{w}_{i}^{\dagger} \mathbf{w}_{i}-\alpha\left(\sum_{\forall j \in \mathcal{G}_{G+1}} \xi_{j}\right) \\
\text { s.t. }(C 1)_{a},(C 1)_{b},(C 2)_{a},(C 2)_{b}^{\star} .
\end{aligned}
$$

We observe that $(P 5)$ is a non-convex problem, specifically when the optimization variables are considered jointly. However, an alternating parameter optimization based on an iterative process may be employed to seek a suitable solution of $(P 5)$. The proposed solution is discussed in later section.

\subsection{Semi-definite programming (SDP)}

Herein, we define $\mathbf{w}=\left[\begin{array}{l}\mathbf{w}_{1}^{\mathrm{T}} \\ \mathbf{w}_{2}^{\mathrm{T}} \ldots \mathbf{w}_{G+1}^{\mathrm{T}}\end{array}\right]^{\mathrm{T}}, \mathbf{W}_{i}=\mathbf{w}_{i} \mathbf{w}_{i}^{\dagger}$, and $\mathbf{H}_{i}=\mathbf{h}_{i} \mathbf{h}_{i}^{H}$. With the help of these notations, we transform $(P 4)$ using the semi-definite programming (SDP) with further utilization of the semi-definite relaxation (SDR) as follows

$$
\begin{aligned}
(P 6): \min _{\substack{\left\{\mathbf{W}_{i}\right\}_{i=1}^{G+1}, \alpha,\left\{\xi_{j}\right\}_{\forall j \in \mathcal{G}_{G+1}}}} \sum_{i=1}^{G+1} \operatorname{Tr}\left\{\mathbf{W}_{i}\right\}-\alpha\left(\sum_{\forall j \in \mathcal{G}_{G+1}} \xi_{j}\right) \\
\text { s.t. }(C 1): \operatorname{Tr}\left\{\mathbf{H}_{i} \mathbf{W}_{z}\right\}-\gamma_{x} \sum_{z \neq y} \operatorname{Tr}\left\{\mathbf{H}_{i} \mathbf{W}_{y}\right\} \\
\geq \gamma_{x}\left(\sigma_{R, x}^{2}+\sigma_{D, x}^{2}\right), \forall x \in \mathcal{G}_{y}, \\
\forall y \in\{1, \ldots, G\}, \forall z \in\{1, \ldots, G+1\}, \\
(C 2): \widehat{\mathcal{A}}\left(\mathbf{W}_{j}\right) \geq \xi_{j} \widehat{\mathcal{B}}\left(\mathbf{W}_{j}\right), \forall j \in \mathcal{G}_{G+1}, \\
(C 3): \mathbf{W}_{k} \succcurlyeq 0, \quad k=\{1, \cdots, G+1\},
\end{aligned}
$$

where $\widehat{\mathcal{A}}\left(\mathbf{W}_{j}\right)=\mathcal{E}^{\prime}\left(1-\nu\left(1+e^{\left(-\lambda\left(\sum_{i=1}^{G+1} \operatorname{Tr}\left\{\mathbf{H}_{j} \mathbf{W}_{i}\right\}\right)+\lambda \mu\right)}\right)\right)$ and $\widehat{\mathcal{B}}\left(\mathbf{W}_{j}\right)=(1-\nu)\left(1+e^{\left(-\lambda\left(\sum_{i=1}^{G+1} \operatorname{Tr}\left\{\mathbf{H}_{j} \mathbf{W}_{i}\right\}\right)+\lambda \mu\right)}\right)$, while the other parameters have same definitions as described before. ${ }^{1}$ It is clear that joint optimization of the intended variables is cumbersome due to the non-convexity of $(P 6)$. Similar as above, an alternating parameter optimization based on an iterative process may be sought to find a suitable solution. The proposed technique is discussed in the following section.

\subsection{Proposed Solution}

In order to solve $(P 5) /(P 6)$, we propose a tri-level alternating parameter (TAP) based iterative algorithm. To proceed, we first fix the values of $\alpha$, and $\left\{\xi_{j}\right\}_{\forall j \in \mathcal{G}_{G+1}}$ in the first level of the TAP algorithm, such that the constraints in $(P 5) /(P 6)$ are satisfied, thereby making the problem convex and tractable with respect to $\left(\left\{\mathbf{w}_{i}\right\}_{i=1}^{G+1},\left\{\varsigma_{j}\right\}_{\forall j \in \mathcal{G}_{G+1}}\right) /\left\{\mathbf{W}_{i}\right\}_{i=1}^{G+1}$ accordingly. In this context, standard convex optimization methods may be employed to obtain suitable solution for the intended parameter(s), i.e., $\left(\left\{\mathbf{w}_{i}\right\}_{i=1}^{G+1},\left\{\varsigma_{j}\right\}_{\forall j \in \mathcal{G}_{G+1}}\right) /\left\{\mathbf{W}_{i}\right\}_{i=1}^{G+1}$. Next, we fix the values of $\left(\left\{\mathbf{w}_{i}\right\}_{i=1}^{G+1},\left\{\varsigma_{j}\right\}_{\forall j \in \mathcal{G}_{G+1}}\right) /\left\{\mathbf{W}_{i}\right\}_{i=1}^{G+1}$ (as obtained from the first level of TAP algorithm) and $\alpha$, to obtain the corresponding solutions of $\left\{\xi_{j}\right\}_{\forall j \in \mathcal{G}_{G+1}}$, using the same process as described in the first level of the proposed TAP algorithm. Thereafter, we set the values of $\left(\left\{\mathbf{w}_{i}\right\}_{i=1}^{G+1},\left\{\varsigma_{j}\right\}_{\forall j \in \mathcal{G}_{G+1}}\right) /\left\{\mathbf{W}_{i}\right\}_{i=1}^{G+1}$ and $\left\{\xi_{j}\right\}_{\forall j \in \mathcal{G}_{G+1}}$, as obtained from the first and second levels of TAP algorithm, respectively, and find the solution for $\alpha$ by employing the same mechanism as mentioned for the two-levels of TAP. Finally, we make use of the solutions of $\alpha$ and $\left(\left\{\mathbf{w}_{i}\right\}_{i=1}^{G+1},\left\{\varsigma_{j}\right\}_{\forall j \in \mathcal{G}_{G+1}}\right) /\left\{\mathbf{W}_{i}\right\}_{i=1}^{G+1}$ and $\left\{\xi_{j}\right\}_{\forall j \in \mathcal{G}_{G+1}}$ obtained from the second and third level of the TAP algorithm

\footnotetext{
${ }^{1}$ It is noteworthy that the non-convex constraint of $\operatorname{rank}\left(\mathbf{W}_{k}\right)=1, k=$ $\{1, \cdots, G+1\}$ is dropped following the standard SDR procedure [18].
} 
Accepted to The international Conference on Acoustics, Speech, \& Signal Processing (ICASSP), Toronto, Canada, 6-11 June 2021.

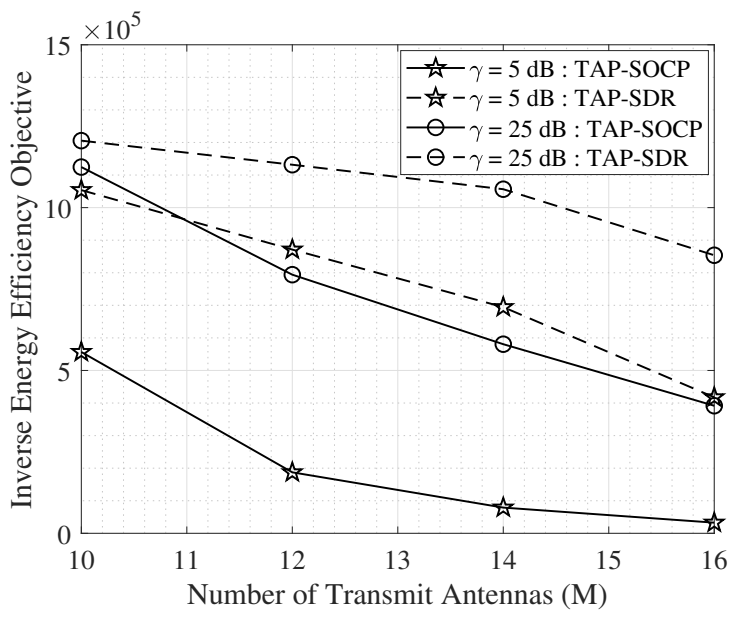

Fig. 1. Energy efficiency optimization with respect to the incremental values of $\mathrm{M}$ and $\gamma$, where $\mathrm{D}=5 \mathrm{~m}$.

and provide them as input for the first level of the algorithm. This iterative process is carried out until a guaranteed convergence of the objective value in $(P 5) /(P 6)$.

From further analysis, the decreasing objective value may be seen at each iterative cycle of the TAP algorithm. Hence, the convergence proof of the algorithm is straightforward, and is omitted for brevity. In terms of the computational complexity, SOCP is naturally an efficient method over the SDR. This is primarily due to the involvement of lesser optimization variables (per iteration) in the former $(\mathcal{O}((G+1)(M+2)))$ over the latter $\left(\mathcal{O}\left((G+1)\left(M^{2}+1\right)\right)\right)$, provided $M \geq(G+1)$.

\section{SIMULATION RESULTS}

In this section, we present the performance benefits of the proposed TAP algorithm under the considered framework targeting the design of a dedicated precoder to serve the set of users with EH demands, in addition to the MC precoding. To solve the simplified convex problems, we make use of the convex programming tool CVX $[19,20]$, with solutions obtained via SEDUMI solver. We assume an ITU-R indoor model (2-floor office scenario) to generate channel realizations with the pathloss exponent given by [21]

$$
\mathrm{PL}(\text { in } \mathrm{dB})=20 \log _{10}(\mathrm{~F})+N \log _{10}(\mathrm{D})+P_{f}(n)-38,
$$

where $\mathrm{F}=2.4 \mathrm{GHz}$ is the operational frequency, $N=30$ is the distance power loss coefficient, $\mathrm{D}=5 \mathrm{~m}$ (unless specified otherwise) is the separation distance between the transmitter and end-user(s), $P_{f}(n)=15+4(n-1)=19 \mathrm{~dB}$ is the floor penetration loss factor, and $n=2$ is the number of floors between the transmitter and the end-user(s). ${ }^{2}$

The transmitter is assumed to be equipped with $M=16$ antennas (unless specified otherwise) while $L=10$ users are distributed within $(G+1)=5$ multicasting groups as follows: $\mathcal{G}_{1}=\left\{\mathcal{D}_{1}, \mathcal{D}_{3}, \mathcal{D}_{4}\right\}, \mathcal{G}_{2}=\left\{\mathcal{D}_{2}, \mathcal{D}_{5}\right\}, \mathcal{G}_{3}=\left\{\mathcal{D}_{6}, \mathcal{D}_{8}\right\}, \mathcal{G}_{4}$

\footnotetext{
${ }^{2}$ Note that an additional antenna gain of $10 \mathrm{~dB}$ is added to take the directivity of the transmit antennas into account.
}

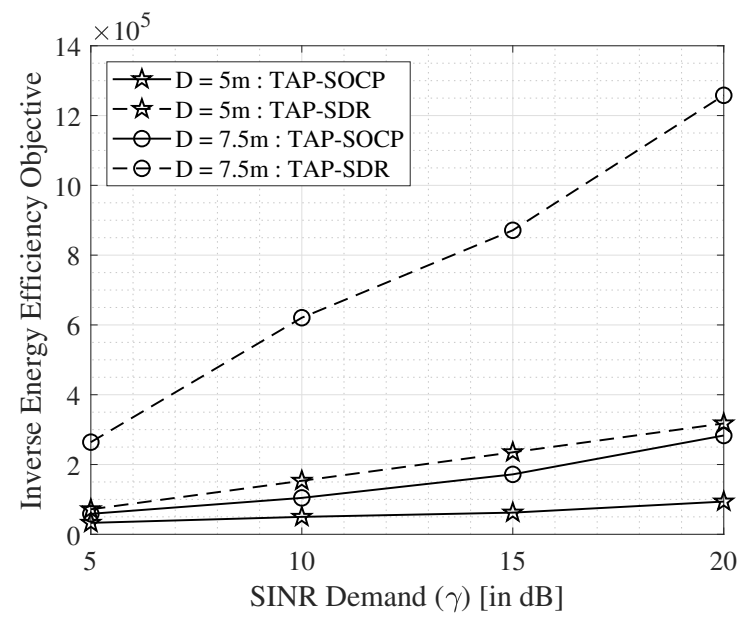

Fig. 2. Energy efficiency optimization with respect to the incremental values of $\gamma$ and $\mathrm{D}$, where $\mathrm{M}=16$.

$=\left\{\mathcal{D}_{7}, \mathcal{D}_{9}, \mathcal{D}_{10}\right\}$, and $\mathcal{G}_{5}=\left\{\mathcal{D}_{1}, \mathcal{D}_{5}, \mathcal{D}_{8}, \mathcal{D}_{10}\right\}$, where $\mathcal{G}_{5}$ is the energy harvesting group of users while the remaining $\left(\mathcal{G}_{1}, \cdots\right.$, $\mathcal{G}_{4}$ ) groups are comprised of information users. We set to $\sigma_{r, i}^{2}$ $=-110 \mathrm{dBW}$, and $\sigma_{d, i}^{2}=-80 \mathrm{dBW}$. The constants corresponding to the non-linear EH circuit are chosen as $\mathcal{E}^{\prime}=2.8 \mathrm{~mJ}, \lambda$ $=1500$, and $\mu=0.0022[15,16]$. For simplicity, we assume $\gamma_{i}=\gamma,\left.\forall i \in \mathcal{G}_{\ell}\right|_{\ell=1} ^{G}$.

In Fig. 1, we plot the inverse energy efficiency objective in $(P 5) /(P 6)$ versus $M$ for different values of $\gamma$ at the corresponding ID users. We observe that the TAP algorithm provides considerable gains via SOCP over SDR for increasing values of $M$. However, when the $\gamma$ is increased from $5 \mathrm{~dB}$ to $25 \mathrm{~dB}$, the objective in $(P 5) /(P 6)$ increases primarily due to the requirement of higher transmit energy in the latter case.

We show in Fig. 2 the effect of increasing $\gamma$ and $\mathrm{D}$ on the inverse energy efficiency objective in $(P 5) /(P 6)$, wherein an increment is seen for both cases. More specifically, the objective values grow non-linearly with the increasing values of $\gamma$ as well as when D is increased from $5 \mathrm{~m}$ to $7.5 \mathrm{~m}$. However, we observe significant benefits of the SOCP-enabled TAP over the SDR-based TAP algorithm.

\section{CONCLUSION}

We considered a precoder design problem for SWIPT-enabled MG-MC systems with heterogeneous wireless user types that are capable of performing ID only, EH only, and joint ID and EH. In this regard, we proposed and formulated an optimization problem to minimize the ratio of total transmit energy to the sum-harvested energy at the intended users, under a constraint on minimum SINR at the MC group of users. In order to solve the above-mentioned non-convex problem, we proposed an algorithm based on Dinkelback method, slackvariable replacement, and SOCP/SDR. In this context, the SOCP-assisted tri-level alternating parameter (TAP) based iterative algorithm was found to perform considerably better than the SDR-based TAP algorithm. 
Accepted to The international Conference on Acoustics, Speech, \& Signal Processing (ICASSP), Toronto, Canada, 6-11 June 2021.

\section{REFERENCES}

[1] Y. B. Zikria, S. W. Kim, M. K. Afzal, H. Wang, and M. H. Rehmani, "5G Mobile Services and Scenarios: Challenges and Solutions," Sustainability, vol. 10, no. $10,2018$.

[2] S. Gautam, et al., "Multigroup Multicast Precoding for Energy Optimization in SWIPT Systems With Heterogeneous Users," IEEE Open Journal of the Communications Society, vol. 1, pp. 92-108, 2020.

[3] S. Gautam, E. Lagunas, S. Chatzinotas, and B. Ottersten, "Wireless Multi-group Multicast Precoding with Selective RF Energy Harvesting," in 2019 27th European Signal Processing Conference (EUSIPCO), A Coruña, Spain, 2019, pp. 1-5.

[4] N. D. Sidiropoulos and T. N. Davidson and, "Transmit beamforming for physical-layer multicasting," IEEE Trans. Signal Process., vol. 54, no. 6, pp. 2239-2251, June 2006.

[5] J. Krivochiza, et al., "FPGA Acceleration for Computationally Efficient Symbol-Level Precoding in MultiUser Multi-Antenna Communication Systems," IEEE Access, vol. 7, pp. 15509-15520, 2019.

[6] M. R. A. Khandaker and K. Wong, "SWIPT in MISO Multicasting Systems," IEEE Wireless Commun. Lett., vol. 3, no. 3, pp. 277-280, June 2014.

[7] M. Alodeh, et al., "Symbol-Level and Multicast Precoding for Multiuser Multiantenna Downlink: A Stateof-the-Art, Classification, and Challenges," IEEE Commun. Surveys Tuts., vol. 20, no. 3, pp. 1733-1757, thirdquarter 2018.

[8] D. Christopoulos, S. Chatzinotas, and B. Ottersten, "Weighted Fair Multicast Multigroup Beamforming under Per-antenna Power Constraints," IEEE Trans. Signal Process., vol. 62, no. 19, pp. 5132-5142, 2014.

[9] A. Bandi, R. Bhavani Shankar Mysore, S. Chatzinotas and B. Ottersten, "Joint User Grouping, Scheduling, and Precoding for Multicast Energy Efficiency in Multigroup Multicast Systems," IEEE Trans. Wireless Commun., pp. 1-1, 2020.

[10] Z. Ding, et al., "Application of smart antenna technologies in simultaneous wireless information and power transfer," IEEE Comm. Mag., vol. 53, no. 4, pp. 86-93, April 2015.

[11] Ö. T. Demir and T. E. Tuncer, "Multi-group multicast beamforming for simultaneous wireless information and power transfer," in 23rd European Sig. Processing Conf. (EUSIPCO), Aug 2015, pp. 1356-1360.
[12] Ö. T. Demir and T. E. Tuncer, "Antenna Selection and Hybrid Beamforming for Simultaneous Wireless Information and Power Transfer in Multi-Group Multicasting Systems," IEEE Trans. Wireless Comm., vol. 15, no. 10, pp. 6948-6962, Oct 2016.

[13] D. Mishra, G. C. Alexandropoulos, and S. De, "Energy Sustainable IoT With Individual QoS Constraints Through MISO SWIPT Multicasting," IEEE Internet Things J., vol. 5, no. 4, pp. 2856-2867, Aug 2018.

[14] S. Gautam, et al., "Weighted Sum-SINR and Fairness Optimization for SWIPT-Multigroup Multicasting Systems With Heterogeneous Users," IEEE Open Journal of the Communications Society, vol. 1, pp. 1470-1484, 2020.

[15] J. Guo, H. Zhang, and X. Zhu, "Theoretical analysis of RF-DC conversion efficiency for class-F rectifiers," IEEE Trans. Microw. Theory Techn., vol. 62, no. 4, pp. 977-985, 2014.

[16] S. Gautam, E. Lagunas, S. Chatzinotas, and B. Ottersten, "Relay Selection and Resource Allocation for SWIPT in Multi-User OFDMA Systems," IEEE Trans. Wireless Commun., vol. 18, no. 5, pp. 2493-2508, 2019.

[17] Milton Abramowitz and Irene A Stegun, Handbook of Mathematical Functions: with Formulas, Graphs, and Mathematical Tables, vol. 55, US Government printing office, 1970.

[18] Mats Bengtsson and Björn Ottersten, Optimal and suboptimal transmit beamforming, CRC press, 2001.

[19] M. Grant and S. Boyd, "CVX: Matlab Software for Disciplined Convex Programming, version 2.1," http://cvxr.com/cvx, Mar. 2014.

[20] Michael Grant and Stephen Boyd, "Graph implementations for nonsmooth convex programs," in Recent Advances in Learning and Control, V. Blondel, S. Boyd, and H. Kimura, Eds., Lecture Notes in Control and Information Sciences, pp. 95-110. Springer-Verlag Limited, 2008, http://stanford.edu/ boyd/graph_dcp.html.

[21] P. Series, "Propagation data and prediction methods for the planning of indoor radiocommunication systems and radio local area networks in the frequency range 900 MHz to $100 \mathrm{GHz}$," Recommendation ITU-R, pp. 1238$7,2012$. 